\title{
Updates in Perioperative Medicine
}

\author{
Suparna Dutta, MD, MPH ${ }^{1 *}$, Steven L. Cohn, MD², Kurt J. Pfeifer, MD³, Barbara A. Slawski, MD, MS³, \\ Gerald W. Smetana, MD', Amir K. Jaffer, MD, MBA'
}

\begin{abstract}
${ }^{1}$ Department of Medicine, Rush Medical College, Chicago, Illinois; ${ }^{2}$ University of Miami Miller School of Medicine, Miami, Florida; ${ }^{3}$ Froedtert Memorial Lutheran Hospital Clinical Cancer Center and Medical College of Wisconsin, Milwaukee, Wisconsin; ${ }^{4}$ Division of General Medicine and Primary Care, Harvard Medical School, Boston, Massachusetts.
\end{abstract}

BACKGROUND: As our surgical population becomes older and more medically complex, knowledge of the most recent perioperative literature is necessary to optimize perioperative care. We aim to summarize and critique literature published over the past year with the highest potential to impact the clinical practice of perioperative medicine.

METHODS: We reviewed articles published between January 2014 and April 2015, identified via MEDLINE search. The final 10 articles selected were determined by consensus among all authors, with criteria for inclusion including scientific rigor and relevance to perioperative medicine practice.

RESULTS: Key findings include: long term $\beta$-blockade should be continued prior to surgery, routine screening with postoperative troponin is not recommended, initiation/continuation of aspirin or clonidine in the perioperative period is not beneficial and may increase adverse outcomes, preoperative diagnosis and treatment of obstructive sleep apnea may reduce risk of postoperative cardiovascular complications, new pulmonary risk indices are available that accurately estimate postoperative pulmonary complications, postoperative atrial fibrillation is associated with increased long-term stroke risk, risk scores such as the $\mathrm{CHADS}_{2}$ (Congestive heart failure, Hypertension, Age $\geq 75$ years, Diabetes Mellitus, previous stroke or transient ischemic attack) are superior to the Revised Cardiac Risk Index in predicting adverse postoperative outcomes for patients with nonvalvular atrial fibrillation, and utilization of bridging anticoagulation comes with a much higher risk of bleeding compared to patients who are not bridged.

CONCLUSIONS: The body of literature reviewed provides important information for clinicians caring for surgical patients across multiple fronts, including preoperative risk assessment, medication management, and postoperative medical care. Journal of Hospital Medicine 2016;11:231236. (C) 2015 Society of Hospital Medicine
Given the rapid expansion of the field of perioperative medicine, clinicians need to remain apprised of the current evidence to ensure optimization of patient care. In this update, we review 10 key articles from the perioperative literature, with the goal of summarizing the most clinically important evidence over the past year. This summary of recent literature in perioperative medicine is derived from the Update in Perioperative Medicine sessions presented at the 10th Annual Perioperative Medicine Summit and the Society of General Internal Medicine 38th Annual Meeting. A systematic search strategy was used to identify pertinent articles, and the following were selected by the authors based on their relevance to the clinical practice of perioperative medicine.

\section{PERIOPERATIVE CARDIOVASCULAR CARE}

Fleisher LA, Fleischmann KE, Auerbach AD, et al. 2014 ACC/AHA guideline on perioperative cardiovascular

\footnotetext{
*Address for correspondence and reprint requests: Suparna Dutta, MD, Rush Medical College, 1717 W. Congress Pkwy, 1029 Kellogg, Chicago, IL 60612; Telephone: 312-942-4200; Fax: 312-342-3568; E-mail: suparna_dutta@Rush.edu

Additional Supporting Information may be found in the online version of this article.

Received: June 23, 2015; Revised: August 20, 2015; Accepted: August 24, 2015

2015 Society of Hospital Medicine DOI 10.1002/jhm.2487

Published online in Wiley Online Library (Wileyonlinelibrary.com).
}

evaluation and management of patients undergoing noncardiac surgery: a report of the American College of Cardiology/American Heart Association Task Force on practice guidelines. Circulation. 2014;130:e278-e333.

\section{Background}

The American College of Cardiology/American Heart Association (ACC/AHA) perioperative guideline provides recommendations for the evaluation and management of cardiovascular disease in patients undergoing noncardiac surgery.

\section{Findings}

The new guideline combines the evaluation of surgeryand patient-specific risk in the algorithm for preoperative cardiovascular evaluation into a single step and recommends the use of 1 of 3 tools: the Revised Cardiac Risk Index (RCRI), ${ }^{1}$ National Surgical Quality Improvement Program (NSQIP) Surgical Risk Calculator, ${ }^{2}$ or the NSQIP-derived myocardial infarction and cardiac arrest calculator. ${ }^{3}$ Estimation of risk is also simplified by stratification into only 2 groups: low risk (risk of major adverse cardiac event $<1 \%$ ) and elevated risk ( $\geq 1 \%$ risk). Coronary evaluation can be considered for patients with elevated cardiac risk and poor functional capacity, but is advised only if the results would alter perioperative management. For example, a patient with very high risk who has evidence of ischemia on stress testing may choose to forego surgery. Preoperative coronary revascularization is 
only indicated for patients meeting criteria in the nonsurgical setting.

For patients with previous percutaneous coronary intervention, the ACC/AHA has not changed its recommendations to optimally delay surgery for at least 30 days after bare-metal stenting and at least 1 year after drug-eluting stent (DES) placement. However, in patients with a DES placed 6 to 12 months previously, surgery can be performed if the risks of surgical delay outweigh the risks of DES thrombosis. After any type of coronary stenting, dual antiplatelet therapy should be continued uninterrupted through the first 4 to 6 weeks and even later whenever feasible. If not possible, aspirin therapy should be maintained through surgery unless bleeding risk is too high.

The guideline recommends perioperative continuation of $\beta$-blockers in patients taking them chronically. Preoperative initiation of $\beta$-blocker therapy may be considered for patients with myocardial ischemia on stress testing or $\geq 3$ RCRI factors and should be started far enough in advance to allow determination of patient's tolerance prior to surgery.

\section{Cautions}

Many recommendations are based on data from nonrandomized trials or expert opinion, and the data in areas such as perioperative $\beta$-blockade continue to evolve.

\section{Implications}

The ACC/AHA guideline continues to be a critically valuable resource for hospitalists providing perioperative care to noncardiac surgery patients.

Wijeysundera DN, Duncan D, Nkonde-Price C, et al. Perioperative beta blockade in noncardiac surgery: a systematic review for the 2014 ACC/AHA guideline on perioperative cardiovascular evaluation and management of patients undergoing noncardiac surgery: a report of the American College of Cardiology/American Heart Association Task Force on practice guidelines.

J Am Coll Cardiol. 2014;64(22):2406-2425.

\section{Background}

Various clinical trials have reported conflicting results regarding the efficacy and safety of perioperative $\beta$ blockers resulting in guideline committees changing their recommendations. Because of questions raised regarding the scientific integrity of the DECREASE (Dutch Echocardiographic Cardiac Risk Evaluation Applying Stress Echocardiography) $-\mathrm{I}^{4}$ and DECREASE-IV $\mathrm{V}^{5}$ trials as well as the dosing of $\beta$-blockers in POISE (PeriOperative Ischemic Evaluation) study, ${ }^{6}$ this systematic review was performed in conjunction with the ACC/AHA guideline update ${ }^{7}$ to evaluate the data with and without these trials.

\section{Findings}

Sixteen randomized control trials (RCTs) $(\mathrm{n}=12,043)$ and 1 cohort study $(n=348)$ were included in the analysis. Perioperative $\beta$-blockers were associated with a reduction in nonfatal myocardial infarction (MI) (relative risk [RR]: 0.69; 95\% confidence interval $[\mathrm{CI}]: 0.58-0.82 ; P<0.001)$ but an increase in bradycardia (RR: 2.61; 95\% CI: 2.18-3.12), hypotension (RR: $1.47 ; 95 \%$ CI: 1.34-1.6), and nonfatal strokes (RR: 1.76 ; 95\% CI: $1.07-2.91 ; P=0.02$ ). The POISE trial was the only one demonstrating a statistically significant increase in stroke.

The major discrepancy between the DECREASE trials and the other RCTs was related to mortality-a reduction in both cardiovascular and all-cause death in DECREASE but an increased risk of all-cause death in the other trials.

\section{Cautions}

Because of its size, the POISE trial heavily influences the results, particularly for mortality and stroke. Including the DECREASE trials reduces the otherwise increased risk for death to a null effect. Exclusion of the POISE and DECREASE trials leaves few data to make conclusions about safety and efficacy of perioperative $\beta$ blockade. Several cohort studies have found metoprolol to be associated with worse outcomes than with atenolol or bisoprolol (which were preferred by the European Society of Cardiology guidelines). ${ }^{8}$

\section{Implications}

Perioperative $\beta$-blockade started within 1 day of noncardiac surgery was associated with fewer nonfatal MIs but at the cost of an increase in hypotension, bradycardia, and a possible increase in stroke and death. Long-term $\beta$-blockade should be continued perioperatively, whereas the decision to initiate a $\beta$-blocker should be individualized. If starting a $\beta$-blocker perioperatively, it should be done $\geq 2$ days before surgery.

Botto F, Alonso-Coello P, Chan MT, et al.; on behalf of The Vascular events In noncardiac Surgery patIents cOhort evaluatioN (VISION) Investigators. Myocardial injury after noncardiac surgery: a large, international, prospective cohort study establishing diagnostic criteria, characteristics, predictors, and 30day outcomes. Anesthesiology. 2014;120(3):564-578.

\section{Background}

Many patients sustain myocardial injury in the perioperative period as evidenced by troponin elevations, but most do not meet diagnostic criteria for MI. Myocardial injury after noncardiac surgery (MINS) is defined as prognostically relevant myocardial injury due to ischemia that occurs within 30 days after noncardiac surgery. This international, prospective cohort study of 15,065 patients $\geq 45$ years old who underwent in-patient noncardiac surgery determined diagnostic criteria, characteristics, predictors, and 30-day outcomes of MINS.

\section{Findings}

The diagnostic criterion for MINS was a peak troponin $\mathrm{T}$ level $\geq 0.03 \mathrm{ng} / \mathrm{mL}$ judged to be due to an ischemic 
etiology. Twelve independent predictors of MINS were identified including age $\geq 75$ years, known cardiovascular disease or risk factors, and surgical factors. MINS was an independent predictor of 30-day mortality (adjusted hazard ratio [HR]: 3.87; 95\% CI: 2.96-5.08). Age $>75$ years, ST elevation, or new left bundle branch block, and anterior ischemic findings were independent predictors of 30-day mortality among patients with MINS.

\section{Cautions}

Although screening high-risk surgical patients without signs or symptoms of ischemia with postoperative troponins will increase the frequency of diagnosing MINS, evidence for an effective treatment has not yet been established. The ACC/AHA guidelines state that routine screening is of uncertain benefit for this reason.

\section{Implications}

Because MINS is common and carries a poor 30-day prognosis, clinical trials are needed to determine when to obtain postoperative troponins and how to prevent and treat this complication. ${ }^{9}$ Some observational data from POISE suggest that aspirin and statins can reduce the risk of 30-day mortality in patients with postoperative MIs.

Devereaux PJ, Mrkobrada M, Sessler DI, et al. for the POISE-2 Investigators. Aspirin in patients undergoing noncardiac surgery. N Engl J Med. 2014; 370(16):1494-1503.

Devereaux PJ, Sessler DI, Leslie K, et al. for the POISE-2 Investigators. Clonidine in patients undergoing noncardiac surgery. N Engl J Med. 2014; 370(16):1504-1513.

\section{Background}

Medical risk reduction with aspirin and other agents in perioperative patients remains controversial. The POISE2 trial is a blinded RCT examining the effects of aspirin and clonidine on outcomes in $>10,000$ noncardiac surgery patients at risk of cardiovascular complications. The aspirin arm of the study included the initiation group and the continuation stratum, as well as placebo. Patients in the clonidine portion of the trial received $0.2 \mathrm{mg}$ of clonidine or placebo daily for the same time periods.

\section{Findings}

The primary outcome was a composite of death or nonfatal MI within 30 days of surgery. Outcomes were similar in patients initiated or continued on aspirin. No difference was seen between aspirin or placebo in the primary outcome $(7.0 \%$ vs $7.1 \%$; HR: $0.86 ; 95 \%$ CI: $0.86-1.15 ; P=0.92)$. There were no differences in rates of MI, venous thromboembolism, or stroke. Major bleeding rates were higher in aspirin versus placebo-treated patients $(4.6 \%$ vs $3.8 \%$; HR: 1.23; 95\% CI: 1.01-1.49; $P=0.04)$.
Clonidine did not alter the composite outcome of death or nonfatal MI $(7.3 \%$ vs $6.8 \%$; HR: $1.08 ; 95 \%$ CI: $0.93-1.26 ; P=0.29$ ). Clinically significant hypotension, bradycardia, and nonfatal cardiac arrest were more common in clonidine-treated patients, although no difference was detected in stroke rates.

\section{Cautions}

Although patients in the trial had cardiovascular risk factors, $<24 \%$ of patients had known coronary artery disease, and $<5 \%$ had coronary stents. Conclusions based on this trial regarding perioperative management of antiplatelet therapy should not include patients with coronary artery stents.

\section{Implications}

Aspirin started before surgery and continued perioperatively did not decrease the rate of death or nonfatal MI but increased the risk of major bleeding. Perioperative management of aspirin needs to be undertaken in the context of cardiac and bleeding risks. Clonidine also did not improve outcomes and increased the risk of bradycardia and hypotension. Current guidelines recommend against using alpha-2 agonists for prevention of perioperative cardiac events ${ }^{7}$; however, patients already on alpha-2 agonists should not stop them abruptly.

\section{PERIOPERATIVE PULMONARY CARE}

Mutter TC, Chateau D, Moffatt M, et al. A matched cohort study of postoperative outcomes in obstructive sleep apnea: could preoperative diagnosis and treatment prevent complications? Anesthesiology. 2014;121(4): 707-718.

\section{Background}

An increasing body of literature associates obstructive sleep apnea (OSA) with an increased risk of postoperative complications. Despite evidence of risk, potential benefits of preoperative diagnosis and treatment of OSA remain unclear.

\section{Findings}

Using databases to identify patients prescribed continuous positive airway pressure (CPAP) therapy, the study compared postoperative outcomes of patients who underwent surgery any time after polysomnography (PSG) and CPAP prescription (diagnosed OSA [DOSA]) and those who had surgery during the 5 years preceding their PSG (undiagnosed OSA [UOSA]). These patients were matched with patients who underwent the same procedure for the same indication and had no insurance claims for PSG or diagnosis of sleep-disordered breathing.

After multivariate analysis, OSA of any type was associated with increased pulmonary complications (odds ratio [OR]: 2.08 ; 95\% CI: 1.35-2.19). However, no significant differences in respiratory outcomes were noted between DOSA patients $(\mathrm{N}=2640)$ and those with UOSA $(\mathrm{N}=1571)$. DOSA patients did have fewer 
cardiovascular complications than UOSA patients (OR: 0.34; 95\% CI: 0.15-0.77). Only severe OSA (apneahypopnea index $>30$ ) was associated with increased pulmonary and cardiovascular complications.

\section{Cautions}

Although this study suggests an association between preoperative diagnosis and treatment of OSA and reduced cardiovascular complications, the results are not definitive due to the inability to control for all confounding variables in a retrospective study utilizing an administrative database.

\section{Implications}

OSA is an important risk factor for postoperative complications, and this study suggests that preoperative treatment with CPAP is associated with reduced risk of cardiovascular complications, particularly in patients with severe OSA. Future controlled trials should focus on the risk-reduction potential of preoperative diagnosis and treatment of OSA.

Mazo V, Sabaté S, Canet J, et al. Prospective external validation of a predictive score for postoperative pulmonary complications. Anesthesiology. 2014;121:219231.

\section{Background}

In 2010, Canet et al. published a novel risk index, the Assess Respiratory Risk in Surgical Patients in Catalonia (ARISCAT) index, to provide a quantitative estimate of the risk of postoperative pulmonary complications (PPCs). ${ }^{10}$

In the current report, Mazo and colleagues studied the ARISCAT index in a broader sample to characterize its accuracy in predicting PPC risk. The ARISCAT index is derived from clinical risk factors: (1) age, (2) preoperative oxygen saturation, (3) respiratory infection in the prior month, (4) anemia, (5) surgical site, (6) duration of surgery, and (7) emergency surgery, with varying weights based on the strength of the association in a multivariable analysis. This score can be calculated via addition of these weighted risk factors, with a score $>45$ equal to high risk for PPC.

\section{Findings}

Examining 5099 patients from 63 European hospitals, the authors' definition of PPC included respiratory failure, pulmonary infection, pleural effusion, atelectasis, pneumothorax, bronchospasm, and aspiration pneumonitis. PPC rates were as follows: low risk $(3.39 \%)$, intermediate risk $(12.98 \%)$, and high risk $(38.01 \%)$. The positive likelihood ratio for PPC among the highest risk group was 7.12. The $\mathrm{C}$ statistic for fit was 0.80 . Observed PPC rates were higher than predicted for the low $(3.39 \%$ vs $0.87 \%)$ and intermediate $(12.98 \%$ vs $7.82 \%)$ risk groups.

\section{Cautions}

The calibration slopes were less than ideal in all subsamples, with the Western European sample performing better than the other geographic areas; suggesting that the coefficients on the ARISCAT index may benefit from recalibration to match specific populations.

\section{Implications}

This is the first major pulmonary risk index that has been externally validated. Its use of readily available clinical information, simplicity, and accuracy in estimating PPC risk make it an important addition to the toolkit during a preoperative evaluation.

\section{PERIOPERATIVE ATRIAL FIBRILLATION/ ANTICOAGULATION}

Gialdini G, Nearing K, Bhave P, et al. Perioperative atrial fibrillation and the long term risk of ischemic stroke. JAMA. 2014;312(6):616-622.

\section{Background}

New-onset atrial fibrillation (AF) is the most common perioperative arrhythmia. ${ }^{11}$ However, little is known regarding the long-term risks of ischemic stroke in patients who develop perioperative AF. This retrospective cohort study examined adults with no preexisting history of AF, hospitalized for surgery, and discharged free of cerebrovascular disease between 2007 and $2011(\mathrm{n}=1,729,360)$.

\section{Findings}

Of the eligible patients, $1.43 \%$ (95\% CI: $1.41 \%-$ $1.45 \%$ ) developed perioperative AF, and $0.81 \%(95 \%$ CI: $0.79 \%-0.82 \%$ ) had a stroke up to 1 year after discharge. Perioperative AF was associated with subsequent stroke after both cardiac (HR: 1.3; 95\% CI: 1.1-1.6) and noncardiac surgery (HR: 2; 95\% CI: 1.72.3). The association with stroke was stronger for perioperative AF after noncardiac versus cardiac surgery $(P<0.001$ for interaction).

\section{Cautions}

This is a retrospective cohort study, using claims data to identify AF and stroke. Data on duration of the perioperative $\mathrm{AF}$ episodes or use of antithrombotic therapies were not available.

\section{Implications}

The association found between perioperative AF and long-term risk of ischemic stroke may suggest that perioperative AF, especially after noncardiac surgery, should be treated aggressively in terms of thromboembolic risk; however, further data will be required to validate this association.

Van Diepen S, Youngson E, Ezekowitz J, McAlister F. Which risk score best predicts perioperative outcomes in nonvalvular atrial fibrillation patients undergoing noncardiac surgery? Am Heart J. 2014;168(1):60-67. 


\section{Background}

Patients with nonvalvular AF (NVAF) are at increased risk for adverse perioperative outcomes after noncardiac surgery. ${ }^{12}$ The RCRI is commonly used to predict perioperative cardiovascular events for all patients, including those with NVAF, though AF is not part of this risk assessment. The goal of this retrospective cohort study was to examine the prognostic utility of already existing NVAF risk indices, including the $\mathrm{CHADS}_{2}$ (Congestive heart failure, Hypertension, Age $\geq 75$ years, Diabetes mellitus, prior stroke or transient ischemic attack), $\mathrm{CHA}_{2} \mathrm{DS}_{2}$-VASc (Congestive heart failure; Hypertension; Age $\geq 75$ years; Diabetes mellitus; Stroke, TIA, or thromboembolism [TE]; Vascular disease; Age 65 to 74 years; Sex category [female]), and $\mathrm{R}_{2} \mathrm{CHADS}_{2}$ (Renal dysfunction, Congestive heart failure, Hypertension, Age, Diabetes, Stroke/TIA) for perioperative outcomes in patients undergoing noncardiac surgery.

\section{Findings}

A population dataset of NVAF patients $(n=32,160)$ who underwent noncardiac surgery was examined, with outcome measures including 30-day mortality, stroke, TIA, or systemic embolism. The incidence of the 30-day composite outcome was $4.2 \%$ and the $\mathrm{C}$ indices were 0.65 for the RCRI, 0.67 for $\mathrm{CHADS}_{2}$, 0.67 for $\mathrm{CHA}_{2} \mathrm{DS}_{2}-\mathrm{VASc}$, and 0.68 for $\mathrm{R}_{2} \mathrm{CHADS}_{2}$. The Net Reclassification Index (NRI), a measure evaluating the improvement in prediction performance gained by adding a marker to a set of baseline predictors, was calculated. All NVAF scores performed better than the RCRI for predicting mortality risk (NRI: $12.3 \%, 8.4 \%$, and $13.3 \%$ respectively, all $P<0.01$ ).

\section{Cautions}

Patients in the highest risk category by RCRI appear to have an unadjusted higher 30-day mortality risk $(8 \%)$ than that predicted by the other 3 scores $(5 \%, 5.6 \%$, and $5 \%$ ), indicating that these risk scores should not completely supplant the RCRI for risk stratification in this population. In addition, the overall improvement in predictive capacity of the $\mathrm{CHADS}_{2}, \mathrm{CHA}_{2} \mathrm{DS}_{2}$-VASc, and $\mathrm{R}_{2} \mathrm{CHADS}_{2}$, although superior to the RCRI, is modest.

\section{Implications}

These findings indicate that the preoperative risk stratification for patients with NVAF can be improved by utilizing the $\mathrm{CHADS}_{2}, \mathrm{CHA}_{2} \mathrm{DS}_{2}$-VASc, or $\mathrm{R}_{2} \mathrm{CHADS}_{2}$ scores when undergoing noncardiac surgery. For patients with NVAF identified as high risk for adverse outcomes, this assessment can be integrated into the preoperative discussion on the risks/benefits of surgery.

Steinberg BA, Peterson ED, Kim S, et al. Use and outcomes associated with bridging during anticoagulation interruptions in patients with atrial fibrillation: findings from the Outcomes Registry for Better
Informed Treatment of Atrial Fibrillation (ORBITAF). Circulation. 2015;131:488-494

\section{Background}

Oral anticoagulation (OAC) significantly reduces the risk of stroke in patients with AF. Many AF patients on long-term anticoagulation undergo procedures requiring temporary interruption of OAC. Although guidelines have been published on when and how to initiate bridging therapy, they are based on observational data. Thus, it remains unclear which patients should receive bridging anticoagulation.

\section{Findings}

This is a US registry of outpatients with AF with temporary interruptions of OAC for a procedure. Of 7372 patients treated with OAC, 2803 overall interruption events occurred in 2200 patients (30\%). Bridging anticoagulants were used in $24 \%(\mathrm{n}=665)$. Bleeding events were more common in bridged than nonbridged patients (5.0\% vs $1.3 \%$; adjusted OR: $3.84 ; P<0.0001)$. The overall composite end point of myocardial infarction, stroke or systemic embolism, major bleeding, hospitalization, or death within 30 days was significantly higher in patients receiving bridging (13\% vs $6.3 \%$; adjusted OR: $1.94 ; P=0.0001)$. This statistically significant increase in the composite outcome, which includes cardiovascular events, is most likely in part secondary to inclusion of bleeding events. The recently published BRIDGE (Bridging Anticoagulation in Patients who Require Temporary Interruption of Warfarin Therapy for an Elective Invasive Procedure or Surgery) trial did not find a statistically significant difference in cardiovascular events between bridged and nonbridged patients. ${ }^{13}$

\section{Cautions}

Although patients who were bridged appear to have had more comorbidities and a higher mean $\mathrm{CHADS}_{2}$ score than patients who were not bridged, it is difficult to determine which population of patients may be high risk enough to warrant bridging, as indicated by current American College of Chest Physicians guidelines, as this was not evaluated in this study

\section{Implications}

The use of bridging anticoagulation was significantly associated with higher overall bleeding and adverse event rates. The BRIDGE trial also found that forgoing bridging anticoagulation decreased the risk of major bleeding in patients with AF and was noninferior to bridging for the prevention of arterial TE. ${ }^{13}$

\section{References}

1. Lee T, Marcantonio E, Mangione C, et al. Derivation and prospective evaluation of a simple index for prediction of cardiac risk of major noncardiac surgery. Circulation. 1999;100:1043-1049.

2. Bilimoria KY, Liu Y, Paruch JL, et al. Development and evaluation of the universal ACS NSQIP surgical risk calculator: a decision aid and informed consent tool for patients and surgeons. J Am Coll Surg. 2013;217(5):833-842. 
3. Gupta PK, Gupta H, Sundaram A, et al. Development and validation of a risk calculator for prediction of cardiac risk after surgery. Circulation. 2011;124:381-387.

4. Poldermans D, Boersma E, Bax JJ, et al. The effect of bisoprolol on perioperative mortality and myocardial infarction in high-risk patients undergoing vascular surgery. Dutch Echocardiographic Cardiac Risk Evaluation Applying Stress Echocardiography Study Group. N Engl J Med. 1999;341(24):1789-1794.

5. Dunkelgrun M, Boersma E, Schouten O, et al; Dutch Echocardiographic Cardiac Risk Evaluation Applying Stress Echocardiography Study Group. Bisoprolol and fluvastatin for the reduction of perioperative cardiac mortality and myocardial infarction in intermediate-risk patients undergoing noncardiovascular surgery: a randomized controlled trial (DECREASEIV). Ann Surg. 2009;249(6):921-926.

6. POISE Study Group, Devereaux PJ, Yang H, Yusuf S, et al. Effects of extended-release metoprolol succinate in patients undergoing noncardiac surgery (POISE trial): a randomised controlled trial. Lancet. 2008;371(9627):1839-1847.

7. Fleisher LA, Fleischmann KE, Auerbach AD, et al. American College of Cardiology; American Heart Association. 2014 ACC/AHA guideline on perioperative cardiovascular evaluation and management of patients undergoing noncardiac surgery: a report of the American College of Cardiology/American Heart Association Task
Force on practice guidelines. J Am Coll Cardiol. 2014;64(22): e77-e137.

8. Kristensen SD, Knuuti J, Saraste A, et al. 2014 ESC/ESA Guidelines on non-cardiac surgery: cardiovascular assessment and management: The Joint Task Force on non-cardiac surgery: cardiovascular assessment and management of the European Society of Cardiology (ESC) and the European Society of Anaesthesiology (ESA). Eur Heart J. 2014;35(35):2383-431.

9. Foucrier A, Rodseth R, Aissaoui M, et al. The long-term impact of early cardiovascular therapy intensification for postoperative troponin elevation after major vascular surgery. Anesth Analg. 2014;119(5): 1053-1063.

10. Canet J, Gallart L, Gomar C, et al. ARISCAT Group: Prediction of postoperative pulmonary complications in a population-based surgical cohort. Anesthesiology. 2010;113:1338-1350.

11. Hollenberg SM, Dellinger RP. Noncardiac surgery: postoperative arrhythmias. Crit Care Med. 2000;28(10 suppl):N145-N150.

12. Bhave PD, Goldman LE, Vittinghoff E, et al. Incidence, predictors, and outcomes associated with postoperative atrial fibrillation after major cardiac surgery. Am Heart J. 2012;164(6):918-924.

13. Douketis JD, Spyropoulos AC, Kaatz S, et al. Perioperative bridging anticoagulation in patients with atrial fibrillation. $N$ Engl J Med. 2015;373(9):823-833. 